\title{
A Method to have Multi-Layer Thermal Insulation Provide Damage Detection
}

\author{
Stanley E. Woodard ${ }^{1}$ \\ NASA Langley Research Center, Hampton, VA 23681 \\ Bryant D. Taylor ${ }^{2}$ \\ Swales Aerospace, Hampton, VA 23681 \\ Thomas W. Jones ${ }^{3}$, Qamar A. Shams ${ }^{4}$ \\ NASA Langley Research Center, Hampton, VA 23681 \\ Frankel Lyons 5 \\ ESCG/Hamilton Sundstrand, Houston, Texas 77058 \\ and \\ Donald Henderson ${ }^{6}$ \\ NASA White Sands Test Facility, Las Cruces, NM 88012
}

\begin{abstract}
Design and testing of a multi-layer thermal insulation system that also provides debris and micrometeorite damage detection is presented. One layer of the insulation is designed as an array of passive open-circuit electrically conductive spiral trace sensors. The sensors are a new class of sensors that are electrically open-circuits that have no electrical connections thereby eliminating one cause of failure to circuits. The sensors are powered using external oscillating magnetic fields. Once electrically active, they produce their own harmonic magnetic fields. The responding field frequency changes if any sensor is damaged. When the sensors are used together in close proximity, the inductive coupling between sensors provides a means of telemetry. The spiral trace design using reflective electrically conductive material provides sufficient area coverage for the sensor array to serves as a layer of thermal insulation. The other insulation layers are designed to allow the sensor's magnetic field to permeate the insulation layers while having total reflective surface area to reduce thermal energy transfer. Results of characterizing individual sensors and the sensor array's response to punctures are presented. Results of hypervelocity impact testing using projectiles of 1-3.6 mm diameter having speeds ranging from 6.7-7.1 km/s are also presented.
\end{abstract}

\section{Introduction}

$\mathrm{T}$ HIS paper presents a design for a multi-layer thermal insulation system that includes detecting and locating micrometeorite penetration of an inflatable habitat shell, such as those proposed for lunar habitats, commercial space hotels and TransHab ${ }^{1-3}$. TransHab is an inflatable/deployable structure, Fig. 1, developed by NASA and ILC/Dover. TransHab was a structure having a hard central core and an inflatable outer shell. The shell had four layers: an internal barrier and bladder, a restraint layer, a micrometeorite/orbital debris shield and an external thermal protection blanket. ${ }^{1}$ Bigelow Aerospace is currently developing a modified version of TransHab to use as

\footnotetext{
${ }^{1}$ Senior Scientist, Research and Technology Directorate/MS 230, and AIAA Associate Fellow

${ }^{2}$ Design Engineer, Systems Engineering Directorate/MS 471

${ }^{3}$ Electronics Engineer, Research and Technology Directorate/MS 493

${ }_{5}^{4}$ Senior Electronics Engineer, Systems Engineering Directorate/MS 238

${ }^{5}$ Test Engineer

${ }^{6}$ Project Leader, Hypervelocity Impact (HVI) Test Facility.
} 
an orbital hotel. The first demonstration of the Bigelow structure is Genesis I that was launched July 12, 2006. Genesis I is an inflatable structure $4.4 \mathrm{~m}$ in length whose diameter in low Earth orbit was expanded from $1.6 \mathrm{~m}$ to $2.54 \mathrm{~m}$. The total usable volume is $11.5 \mathrm{~m}^{3}$. It has a $15 \mathrm{~cm}$ thick multi-layer shell.

Detecting and locating any damage from debris or micrometeorites impacting the shell is critical for the use of inflatable structures since these impacts can cause loss of pressure. Uncontrolled pressure loss results in loss of life. ${ }^{2}$ The first orbital study of micrometeorites was one of 12 experiments launched on Sputnik 3 in May 1958. Project Vanguard was the US attempt to put the first satellite into orbit. There were 25 experiments submitted for the launches. Two of the four experiments assigned "Flight Priority A" were for studying micrometeorite impacts and detection. Methods to detect micrometeorite impacts have included audio measurements, with the impact energy being correlated to the measured volume. Plate capacitor arrays are also used, with the capacitance being reduced after impact. ${ }^{4-6}$

Inflatable habitat designs also require extensive multilayer insulation for thermal control. The multi-layer thermal insulation (MLI) blankets limit heat transfer from internal components and the conductive thermal effects from the space environment. Multi-layer insulation is comprised of multiple layers (sometimes as many as 15) of very thin $(<0.0025 \mathrm{~mm})$ low emittance films such as Mylar or Kapton. The films are typically completely metallized on one or both sides and separated with a spacer material such as Dacron to prevent contact of the adjacent metalized layer. ${ }^{7}$ An example multi-layer insulation is shown in Fig. 2.

The MLI system presented herein has one layer designed as a passive sensor array for detecting and locating micrometeorite and debris penetration. Each sensor in the array is an open-circuit electrically conductive spiral trace having no electrical connections that can store electrical and magnetic energy. ${ }^{8,9}$ The significance of the design is that many circuits fail because their connections are damaged or not properly connected. The sensor design presented in this paper inherently eliminates this type of failure. The sensor is also very easy to manufacture. This class of sensors was conceived as a result of this investigation and the first sensor application was to detect damage resulting from surface penetration; however, the sensors can be designed for many measurement applications. Oscillating magnetic fields are used to power the sensors and once electrically active, the sensors respond with their own oscillating magnetic fields. ${ }^{10}$ The frequencies of the magnetic fields are indicative of whether any damage has occurred to the array. When the continuous metalized layers are used, the sensor response and the metalized layers are magnetically coupled resulting in the sensor response strength being attenuated too low to be measured. The coupling was attributable to eddy currents in the metalized film produced by the magnetic field of the sensor. To reduce the amount of attenuation, the remaining MLI layers presented in this paper were designed as a staggered pattern of small copper traces to reduce the magnetic coupling between the sensor response and the MLI metalized film. The modified MLI layers resulted in the sensors' magnetic field response amplitudes being more than sufficient for interrogation

Following this introduction will be a discussion of the design and testing of a sensor designed for damage/penetration detection that includes a brief overview of the method for powering and interrogating it. Measurement results that show how individual sensors respond to damage follow. Penetration testing of the damage detection sensor arrays ( 3 rows of sensors having 3 sensors in each row) is then presented. Two arrays were tested at the NASA White Sands Hypervelocity Impact Facility using projectiles from 1.0 to $3.6 \mathrm{~mm}$ at speeds ranging from 6.7 to $7.1 \mathrm{~km} / \mathrm{s}$. The hypervelocity impact test results are followed by the design and use of the modified nonsensor array MLI layers.

\section{Sensor Design and Testing}

The development of the sensor that was to be used with the interrogation system described in Ref 10 resulted in a new class of sensors for which this is the first application. ${ }^{8,9}$ This class of sensors is different from all previous sensors in that these sensors are electrically open circuits that have no electrical connections. Resistive, capacitive or inductive sensors are electrically connected to a power source and to some form of acquisition equipment that correlates a measurand to the resistance, capacitance or inductance in the circuit, respectively. Sensors using wireless telemetry require the sensor to be electrically connected with the wireless telemetry circuit. Radio frequency identification (RFID) circuits provide telemetry and power but they require a sensor to be electrically connected to the RFID circuit. Previous magnetic field response sensor designs are inductors electrically connected to capacitors that are powered and interrogated using methods discussed in Ref 10. 
The base design of the sensor presented herein is an open-circuit electrically conductive spiral trace. The sensor is shown in Fig. 3. A schematic of the sensor cross-section A-A' is shown in Fig. 3b that illustrates the capacitance between neighboring sensor traces. ${ }^{11}$ When the sensor is made electrically active, it creates a magnetic field along its trace and an electric field between contiguous traces. Its physical behavior when electrically active is similar to that of an inductor (storage of a magnetic field) electrically connected to a capacitor (storage of an electric field) in that it will produce a harmonic magnetic field of its own. In a manner similar to magnetic field response sensors, the changes in frequency, amplitude and bandwidth of the magnetic field produced by the sensor can be correlated with one or more measurands. The only difference is that the new class of sensors incorporates electrically open circuits that do not need a single electrical connection. The open-circuit electrically conductive spiral trace sensors can also be considered as magnetic field response sensors. The sensor geometry or dielectric coatings can be tailored for many different measurement applications.

An impact detection sensor must be able to determine if the surface has been punctured with the hole having at least some prescribed minimum hole or tear size and the sensor must produce a response to interrogation before and after a puncture event. The sensor can be sized geometrically to identify a puncture greater than a predetermined size. For this investigation, the puncture size was $2 \mathrm{~mm}$. The sensor was designed as a square spiral trace. The spacing between contiguous traces was approximately $0.13 \mathrm{~mm}$. The trace width was approximately $2 \mathrm{~mm}$. The sensor resonant frequency was approximately $9.8 \mathrm{MHz}$ for a $15.25 \mathrm{~cm}$ by $15.25 \mathrm{~cm}$ spiral trace size.

\section{A. Method of Powering and Interrogating Sensors}

The magnetic field response acquisition method is used to supply power to the sensors via Faraday induction, and to interrogate the sensor using a "transmit-receive-compare" technique. ${ }^{10}$ A detailed discussion of the method is given in Ref. 10. A series of increasing oscillating magnetic field harmonics is transmitted from an antenna. Each harmonic corresponds to a discrete measurement value. The frequency resolution for the series of harmonics corresponds to the measurement resolution of the physical property. At each harmonic, the sensor is electrically excited as a result of Faraday induction. Once electrically active, the sensor responds with a harmonic magnetic field whose frequency is the resonant frequency of the sensor. The resonant frequency corresponds to the amount of total sensor inductance and capacitance. The antenna ceases transmission so that the sensor magnetic field response can be received by it or another antenna. The sensor response to each transmitted harmonic is compared to the response created by the prior transmitted harmonic. If the response amplitude is higher than the previous one, the new amplitude and the frequency of the harmonic that produced it are stored in memory. The next harmonic is then transmitted by the antenna. The repetition of "transmit-receive-compare" is continued until the transmitted frequency that produces the highest sensor response amplitude is identified. The frequency producing the highest response is the frequency nearest in value to the sensor's resonant frequency. This method of powering the sensor and acquiring the measurement from the sensor does not require a physical connection to a power source or to data acquisition hardware. The method is applicable to any sensor whose resonant frequency is dependent upon having inductance and capacitance.

\section{B. Preliminary Testing of Sensors}

Testing of individual $15.25 \mathrm{~cm}$ by $15.25 \mathrm{~cm}$ sensors has demonstrated that each sensor can be used to identify at least three damage locations per sensor. Preliminary tests show repeatable results. Each sensor was measured to establish its baseline frequency. Three locations were used to gage damage. A template was used to produce consistent locations. A $1.00 \mathrm{Vp}$ signal from a Wavetek $100 \mathrm{MHz}$ Frequency Synthesize Arbitrary Waveform Generator Model 395 electrically connected to an antenna was used to electrically excite each sensor. The antennasensor separation distance was maintained at approx. $7.62 \mathrm{~cm}$ so that the same excitation energy would be used for all tests. The excitation frequency was tuned to that of the sensor. A NASA Langley developed system was used to regulate the antenna transmission and reception. An Agilent E4440A 3-26.5 GHz Spectrum Analyzer was used to measure the response from each sensor. All frequencies shown are in MHz. Figs. 4a and $4 \mathrm{~b}$ show damaged sensors. The sensor in Fig. $4 \mathrm{a}$ was damaged with a $2 \mathrm{~mm}$ hole so that the complete electrical trace would be broken. The response frequency before and after damage are 9.96 and $11.50 \mathrm{MHz}$, respectively. A $2.5 \mathrm{~cm}$ by $2.5 \mathrm{~cm}$ hole was placed in the sensor in Fig. 4b. The response frequency shifted from $10.8 \mathrm{MHz}$ to $13 \mathrm{MHz}$. A larger $4.0 \mathrm{~cm}$ by 4.0 $\mathrm{cm}$ hole produced a response shift from $10.8 \mathrm{MHz}$ to $14.8 \mathrm{MHz}$. The damage to each sensor resulted in two inductively coupled concentric sensors with the damage location being the end of one sensor and the beginning of the other sensor. This was verified by cutting away the inner circuit from the outer circuit and then removing it. When the two were separated, their resonant frequencies were higher than when they were together. 
Figs. 5a and 5b show sensors that had two and three punctures, respectively. The holes are numbered in order of puncturing the sensor. In both cases, the lowest frequency of each new set of inductive coupled concentric circuits changed. In Fig. 5a, the sensor response frequency changed from its undamaged baseline of $9.90 \mathrm{MHz}$ to 11.28 $\mathrm{MHz}$ after a $2 \mathrm{~mm}$ hole was placed along its perimeter. When a second $2 \mathrm{~mm}$ hole was placed in its middle, the lowest response frequency changed to $12.10 \mathrm{MHz}$. Three cuts were made in the sensor of Fig. $5 \mathrm{~b}$. The undamaged response of $9.87 \mathrm{MHz}$ was changed to 9.83 after the first hole and then to 10.37 and 12.17 after holes 2 and 3, respectively.

\section{Damage Detection Array Layer}

Nine sensors ( 3 columns by 3 rows) were used to form the damage detection array layer of the multi-layer insulation. Spacing between the sensors was $0.13 \mathrm{~mm}$. Two arrays are shown in Fig. 6 . Nine open-circuit electrically conductive spiral trace sensors were directly deposited onto a non-conductive substrate. No electrical connections were used between sensors on the array. Eliminating the connection facilitates scaling the method to a larger surface area. The distribution of sensors provided sufficient coverage of the reflective metallic coating to serve as a thermal protection layer. Use of the array for thermal protection will be discussed in a subsequent section of this paper.

Preliminary testing of the array consisted of securing nine individual sensors in the array using Kapton tape at their corners, Fig. 6a. This allowed one sensor that was previously damaged, Fig. 4a, to be placed at different locations in the array. Tests were performed to ascertain how well the shift in response frequencies could be used to determine where in the array a sensor had been damaged. These tests were also used to verify the final array design, Fig. 6b.

During testing, a $1.00 \mathrm{Vp}$ signal at $8.0 \mathrm{MHz}$ from a Wavetek $100 \mathrm{MHz}$ Frequency Synthesize Arbitrary Waveform Generator Model 395 electrically connected to an antenna was used to electrically excite each sensor. The antenna was placed approx $7.62 \mathrm{~cm}$ from the face of each sensor. A laser was fixed to the antenna in a manner that allowed its beam to be used for positioning the antenna consistently. Each sensor's response measurement was taken with laser targeted at the center of each sensor's spiral trace. An Agilent E4440A 3-26.5 GHz Spectrum Analyzer was used to measure the response from each sensor.

Results of the preliminary testing are presented in the Tables 1-3. Each table has 9 cells representing each sensor in the array. In the cells are one or more measured magnetic field response frequencies in MHz. The first table contains measured frequencies when there was no damage to the array. The proximity of the sensors to each other results in inductive coupling. Table 1 would also serve as a baseline. In some cells, more than one frequency is measured due to inductive coupling. In Table 2 are results from placing a damaged sensor at the second column and second row of the array. The damaged sensor had a single $2 \mathrm{~mm}$ puncture along its outer portion. Its resonant frequency when not in proximity to other sensors was $11.50 \mathrm{MHz}$ with the puncture (Fig. 4a). Six of the nine cells were examined. When placed in the array, all frequencies of all sensors were shifted. Location of the damage location could be ascertained by identifying which sensor in the array had the highest resonant frequency. For example, the $12.00 \mathrm{MHz}$ resonance measured at the second row, second column cell is the highest resonant frequency. Response of the sensors bordering near the damage location were also affected more. This could serve as a means to pinpoint damage. In the second damage array test (Table 3), the damaged sensor was placed in first row and last column of the array. Note that it also had the highest frequency $(11.7 \mathrm{MHz})$ in its response. Five cells were interrogated to verify consistency of results. The primary reason for doing the tests were to ascertain if the frequency characteristics along the array can be used to locate damage. Location of damage was determined by identifying the highest frequency of all the sensors. The tests done for Table 2 and 3 were repeated using a damaged sensor having an $11.39 \mathrm{MHz}$ response when not damaged. The results were very consistent with the highest relative error between any two corresponding sensors being $1.2 \%$ and $1.9 \%$ for the tests of Table 2 and 3 , respectively.

\begin{tabular}{|l|l|l|}
\hline 9.50 & $8.03,8.73,9.23,9.93$ & $9.43,9.93$ \\
\hline $8.73,9.33,10.07$ & $8.07,9.00,9.87,11.40$ & $8.80,9.17,10.17$ \\
\hline 9.50 & $8.60,9.07,9.90,11.43$ & 9.87 \\
\hline
\end{tabular}

Table 1 Measured frequencies (MHz) in respective sensor position in array when all sensors are undamaged. 


\begin{tabular}{|l|l|l|}
\hline 8.60 & $8.77,9.37,10.20$ & \\
\hline & $\begin{array}{l}10.20,12.00 \\
\text { *damaged sensor* }\end{array}$ & \\
\hline $8.70,9.53,10.23$ & $9.27,10.10$ & \\
\hline
\end{tabular}

Table 2 Measured frequencies $(\mathrm{MHz})$ in respective sensor position in array when center sensor (position $(2,2)$ ) has been damaged.

\begin{tabular}{|l|l|l|}
\hline $8.83,9.30,9.77$ & 9.80 & $\begin{array}{l}9.77,10.83,11.77 \\
\text { *damaged sensor* }\end{array}$ \\
\hline & $8.40,9.33,9.83,10.90$ & 9.77 \\
\hline 9.77 & & \\
\hline
\end{tabular}

Table 3 Measured frequencies $(\mathrm{MHz})$ in respective sensor position in array when sensor in upper right corner (position $(1,3)$ ) has been damaged.

\section{Hypervelocity Impact Testing}

Hypervelocity impact tests were performed at the White Sands Test Facility to simulate micrometeoroid and orbital debris impacts on an inflatable habitat. Two test articles were used to test the sensor arrays described in this paper and five other detection concepts developed by other investigators. The other concepts used capacitive sensing (damage would change capacitive plate area), piezoelectric accelerometer (impact creates a charge via the direct piezoelectric effect - a directed pressure produces a voltage) array, an acoustic emission sensor and a radio frequency pulse detection system (measure RF emitted during impact). The goal of each sensing system was to detect and locate damage to the habitat micrometeoroid and orbital debris shield. A test article was developed to emulate three shielding layers. A schematic of the sensor concept placement is shown in Fig. 7a. Ten impact tests were performed. In tests 1-5, Langley sensor array No. 2 was placed on layer 1. Langley sensor array No. 5 was placed on layer 2 for tests 6-10. Projectile diameter, mass, velocity and impact location on test articles is presented in Table 4. All projectiles were less than $3.6 \mathrm{~mm}$. The primary objective of the testing at White Sands was to determine if the array could survive multiple damage events and still be interrogated afterwards. Other testing goals were to 1) understand the effect of damage on sensor operations 2) understand the effect of various size energy impacts 3) understand the variability between uses of sensor on exterior versus interior side of the inflatable habitat shield.

The first test article is shown in Fig. 7b. Aluminum frames were used to support the shielding layers. The 0.50 caliber chamber was used. A schematic of the test chamber is shown in Fig. 8a. A projectile is propelled as follows. At one end of a pump tube, a first-stage of smokeless powder is ignited. The exploding charge results in a rapidly expanding gas that moves a piston in the chamber breech. The piston rapidly compresses a light gas column (e.g., hydrogen) as it moves to the other end of the tube where there is a tapered-bored high pressure coupling that stops the piston. Mounted in the coupling is a sabot that holds a projectile. The high pressure gas breaks the burst disk causing the sabot and the projectile to be propelled down the launch tube. In the expansion chamber, the gas pressure is reduced and the sabot separates into two pieces in the expansion chamber as the projectile moves toward the target. Arresting plates in the expansion chamber prevent the sabot pieces from hitting the target. The frames were then attached to a support that was fastened to the bottom of the target chamber as shown in Fig. 8b.

The layers having the Langley sensor array for test articles 1 and 2 are shown in Figs. 9a and 9b, respectively after all impact tests were complete. Impact locations are annotated on the first test article. Sensors 7, 1, 5, 3 and 9 are located at impact locations 1,2, 3, 4 and 5, respectively. All five projectiles from test 1-5 penetrated the first layer of test article 1 (LaRC array No. 2). The resulting damage to sensor array No. 2 were approximately round $0.4,0.5,0.8,0.8$ and $0.9 \mathrm{~cm}$ dia. holes at impact locations $1,2,3,4$ and 5, respectively. Only the last three larger diameter projectiles penetrated the second layer of the second test article. Damage was more severe to array No. 5 placed in the second test article. Array No. 5 is shown in Fig. 10 after testing. Sensor 5 (location 3) had a $6.3 \mathrm{~cm}$ rip. A hole $5.6 \mathrm{~cm}$ by $0.8 \mathrm{~cm}$ with a $4.6 \mathrm{~cm}$ rip and smaller rips were found on sensor 3 (location 4). Damage to sensor 9 (location 5) consisted of a $1.8 \mathrm{~cm}$ by $1.8 \mathrm{~cm}$ hole with a $3.4 \mathrm{~cm}$ rip and $3.6 \mathrm{~cm}$ rip. 


\begin{tabular}{|c|c||r||l|l|}
\hline \multicolumn{1}{|c|}{ Test } & \multicolumn{1}{|c|}{$\begin{array}{c}\text { Projectile } \\
\text { Doiameter }\end{array}$} & \multicolumn{1}{c|}{$\begin{array}{c}\text { Projectile } \\
\text { Mass }\end{array}$} & $\begin{array}{l}\text { Actual } \\
\text { Projectile } \\
\text { Velocity }\end{array}$ & $\begin{array}{l}\text { Impact } \\
\text { Position }\end{array}$ \\
\hline 1 & $1.0 \mathrm{~mm}$ & $0.00146 \mathrm{~g}$ & $7.1 \mathrm{~km} / \mathrm{s}$ & 1 \\
\hline 2 & $1.6 \mathrm{~mm}$ & $0.00597 \mathrm{~g}$ & $6.9 \mathrm{~km} / \mathrm{s}$ & 2 \\
\hline 3 & $2.8 \mathrm{~mm}$ & $0.03234 \mathrm{~g}$ & $6.85 \mathrm{~km} / \mathrm{s}$ & 3 \\
\hline 4 & $3.2 \mathrm{~mm}$ & $0.04732 \mathrm{~g}$ & $6.96 \mathrm{~km} / \mathrm{s}$ & 4 \\
\hline 5 & $3.6 \mathrm{~mm}$ & $0.06556 \mathrm{~g}$ & $6.7 \mathrm{~km} / \mathrm{s}$ & 5 \\
\hline 6 & $1.0 \mathrm{~mm}$ & $0.00145 \mathrm{~g}$ & $7.0 \mathrm{~km} / \mathrm{s}$ & 1 \\
\hline 7 & $1.6 \mathrm{~mm}$ & $0.00597 \mathrm{~g}$ & $7.1 \mathrm{~km} / \mathrm{s}$ & 2 \\
\hline 8 & $2.8 \mathrm{~mm}$ & $0.03233 \mathrm{~g}$ & $7.0 \mathrm{~km} / \mathrm{s}$ & 3 \\
\hline 9 & $3.2 \mathrm{~mm}$ & $0.04720 \mathrm{~g}$ & $6.7 \mathrm{~km} / \mathrm{s}$ & 4 \\
\hline 10 & $3.6 \mathrm{~mm}$ & $0.06556 \mathrm{~g}$ & $6.7 \mathrm{~km} / \mathrm{s}$ & 5 \\
\hline
\end{tabular}

Table 4 Projectile size, mass, velocity and impact position

The first array was not interrogated during tests 1-5. During tests 6-10, an antenna was placed in proximity to sensor 8 and interrogated during impact events 6-10. The piezo film that was used for another damage detection concept had a metallic backing that attenuated the LaRC sensor response during testing so that it was not possible to perform real-time analysis of the damage during the test. The sensor response was sufficient enough that even with the metallic film, all damage events during testing could be identified by changes to frequency response of the sensor in proximity to the interrogation antenna. Due to the inductive coupling of the sensors, only one sensor of the array needed to be interrogated to identify a damage event for any sensor.

Baseline responses prior to impact testing and after all tests were complete are shown for sensor 9 of array No. 2 (impact tests 1-5) and sensor 3 of array No. 5 (impact tests 6-10) in Figs. 11 and 12, respectively. In Fig. 11, the second peak frequency increased by $0.25 \mathrm{MHz}$ and the response amplitude decrease $5-9 \mathrm{dBm}$ beyond $13 \mathrm{MHz}$ as a result of impact tests 1-5. Sensor 3 of Array 5 had a more pronounced change to its response. The first two response peaks were reduced over $12 \mathrm{dBm}$ in magnitude. The third peak frequency increased by $0.5 \mathrm{MHz}$ while its magnitude decreased by more than $20 \mathrm{dBm}$. Peaks 4 and 5 have shifted by over $1 \mathrm{MHz}$ each.

Because the piezo layers prevented real-time analyses of the sequential damage, the post-test damage observed on array No. 5 was replicated sequentially on array No. 6 back at Langley. Figs. 13 - 15 present the responses measured at sensors 5, 3 and 9 as a result of sequential damage to sensor 5, 3 and 9 of array No. 6 that replicated the sequence of damage from the hypervelocity impact tests. The response of each sensor changed as a result of damage to it. In addition to response changes resulting from the damage to itself, each sensor's response changed when other sensors in the array were damaged. The response changes were more pronounced at the damaged sensor. The sensor that was damaged had largest frequency shifts and largest changes in area under the frequency response curve. The change in the area under response curve is proportional to the Euclidean norm of the difference between response magnitude before and after a damage event. 
If the vector of a sensor response magnitude before a damage event is $x_{i}=\left(x_{1 i}, x_{2 i}, \ldots, x_{n i}\right)$ then after the damage event the response magnitude will be $x_{i+1}=\left(x_{1(i+1)}, x_{2(i+1)}, \ldots, x_{n(i+1)}\right)$ and the Euclidean norm, $\|x\|$, of the magnitude difference is

$$
\|x\|=\sqrt{\left(x_{1(i+1)}-x_{1 i}\right)^{2}+\left(x_{2(i+1)}-x_{2 i}\right)^{2}+\ldots+\left(x_{n(i+1)}-x_{n i}\right)^{2}}
$$

Table 5 presents the Euclidean norms from three damage events occurring sequentially at sensors 5,3 and 9 using responses measured at sensors 5,3 and 9. Each row of the table represents a new damage event. Comparison of Figs. 13a, 14a and 15a, shows that the largest shifts in resonant frequency when sensor 5 was damaged are those measured at sensor 5. The Euclidean norms for this event are in the first row of the table. The largest norm of 194.5 was from the response of sensor 5. . Each damage event for any sensor on the array created a new response baseline for each sensor. The next damage event was damage to sensor 3. Comparison of Figs. 13b, 14b and 15b shows the largest resonant frequency shifts resulting from damage to sensor 3 are in the response measured at sensor 3 . The Euclidean norm from the second damage event, row 2 of Table 5, is larger at sensor 3 The last row of Table 5 shows the results from the last damage event. The largest norm is from the change in the response of sensor 9 .. The inductive coupling of the sensors also served as a means of telemetry. The coupling allowed damage to occur at one sensor and be detected at another. To locate damage, two independent methods can be used. The first method is to identify the sensor having the largest shift in its resonant frequencies. The second method is to identify the sensor with the largest Euclidean norm produced using the change in response magnitude. During the impact tests at White Sands Test Facility, an antenna placed at sensor 8 allowed real-time indications of damage to sensor 3, 5 and 9.

\begin{tabular}{|l|l|l|l|}
\hline \multirow{2}{*}{$\begin{array}{c}|c| \\
\text { Damage Event } \\
\text { Location }\end{array}$} & Sensor 5 response & Sensor 3 response & Sensor 9 response \\
\cline { 2 - 4 } & & & \\
\hline Sensor 5 & 194.5 & 116.8 & 139.2 \\
\hline Sensor 3 & 57.0 & 198.5 & 56.4 \\
\hline Sensor 9 & 57.1 & 37.8 & 133.0 \\
\hline
\end{tabular}

Table 5 Euclidean norm of response difference before and after a damage event.

\section{Application of Sensor Array for Thermal Protection}

In a non-gaseous environment, only heat transfer due to conductive and radiative processes needs to be considered for spacecraft thermal control. Ref. 7 provides a detailed discussion on spacecraft thermal control. Multilayer insulation (MLI) is commonly used to prevent heat transfer. ${ }^{7}$ Heat loss occurs as the heat generated by an instrument or subsystem propagates out. External heat transfer results from rocket plumes or thermal changes due terminator crossing (orbital spacecraft). A secondary MLI function is that of protecting against damage from micrometeorites, atomic oxygen, electron charge accumulation, and rocket plume impingement. ${ }^{7}$ The sensor array previously described can serve as one of the thermal layers of the MLI. Each MLI layer is a low-emittance film. One example of MLI is multiple layers of embossed $0.25 \mathrm{~mm}$ thick Mylar with a vacuum-deposited aluminum on one side. ${ }^{7}$ The aluminized layers serve as a means of reducing radiative heat transfer. Aluminum is electrically conductive. If an array of rectangular spiral traces of Aluminum were deposited directly on one of the Mylar layers in lieu of a continuous deposition, that layer could serve two functions - micrometeorite detection and thermal control.

To develop a thin-film substrate as a reflective surface, the bonding surfaces are cleaned and dried with isopropyl alcohol. To achieve the desired results, it may be necessary to seal or prime the substrate. Thin film bond strength is dependent upon the amount of adhesion to the surface contact. The applied pressure develops better adhesive contact, consequently better bond strength. At room or low temperature, about $50 \%$ of the ultimate strength is achieved within half an hour and might increase bond strength to $90 \%$ after 24 hours of curing. The vacuum 
deposited and thermal controlled coatings have been optimized on a variety of lightweight flexible substrates. The maximum reflectivity of the thin film can be achieved by two steps. First, if thin films are in trace form (like traces of an inductor), the films are deposited in convex form. When these films are subjected to heat treatment, it causes them to assume a rounded shape along the traces. Afterwards a very thin layer of aluminum or silver is deposited which makes these films highly reflective. The absorptive and reflective properties of the films depend upon deposited materials. Silver, aluminum, and to some extent copper are the most popular metals to achieve flexible optical surfaces.

A method to estimate the insulation effectiveness of MLI is to determine its effective emittance. Effective emittance, $\varepsilon$, can be measured experimentally at gas pressures less than $10^{-5}$ torr or calculated as

$$
\varepsilon=\frac{Q}{A \sigma\left(T_{H}^{4}-T_{C}^{4}\right)}
$$

$A, Q, \sigma, T_{C}$ and $T_{H}$ are the reflective surface area $\left(\mathrm{m}^{2)}\right.$, net heat transferred in units of $\mathrm{W}$, Stefan-Boltzman constant in units of $\mathrm{W} / \mathrm{m}^{2}-\mathrm{K}^{4}$, and, cold and hot boundary temperatures in degree Kelvin, respectively. ${ }^{13}$ When the sensors are deposited onto a MLI layer, a portion of the surface is no longer reflective because of the spacing between contiguous traces. Effective emittance is reduced proportional to the loss of reflective area. A first order approximation of the effective area $A_{\text {eff }}$ is to scale the area without traces, $A$, by the ratio of the sensor trace width, $w_{i}$, to the combined sensor width and spacing width, $w_{s}$. Therefore

$$
A_{e f f}=\left(\frac{w_{i}}{w_{i}+w_{s}}\right) A
$$

The effective emittance is now

$$
\varepsilon=\frac{Q}{A_{e f f} \sigma\left(T_{H}^{4}-T_{C}^{4}\right)}
$$

Using the sensor dimensions given earlier, $A_{\text {eff }}=0.94 A$ resulting in a $6 \%$ increase of effective emittance for the layer having the sensor array. Because this layer is one of many layers, the overall change in MLI insulative properties should be negligible.

The circuit design shown in Fig. 16 is used for the other thermal insulation layers. The circuit design is necessary to prevent the sensor response from being absorbed in the continuous metallic surface layers. To operate as an integral part of the multi-layer shell, the sensors must maintain sufficient signal response amplitude to assure a measurable response. During developmental testing it was determined that a magnetic coupling between the sensor and the MLI metalized film existed and resulted in an attenuated response from the sensor. The coupling was attributable to eddy currents in the metalized film produced by the magnetic field of the inductor. These currents flow in a direction opposite to that of the sensor current and have the effect of decreasing the inductance and signal response. This phenomenon is accurately described by Lenz's law; The electromotive force (emf) induced in an electric circuit always acts in such a direction that the current it drives around a closed circuit produces a magnetic field which opposes the change in magnetic flux. For our application, eddy currents were induced in the metallic surface of the MLI layer by the magnetic field of the sensor. The induced current flowed in a direction opposite to that of the inductor current. ${ }^{12}$ This resulted in a negative mutual coupling between the currents, effectively reducing the magnetic field response.

The effect is similar to a transformer where the primary and secondary circuits represent the sensor and the metalized surface, respectively. The circuit diagram in Fig. 16 represents this effect. The induced current in the 
metalized film will impose a counter electromotive force on the sensor. This effect can be accounted for by adding a reflected impedance Zr given in Eq. (4) in series with the primary circuit (Eq. (5)).

$$
\begin{gathered}
Z_{R}(j w)=\frac{\omega^{2} M^{2}}{R_{2}+j \omega L_{2}} \\
Z_{\text {input }}(j \omega)=R_{1}+j \omega C_{1}+j \omega L_{1}+Z_{R}
\end{gathered}
$$

Note that the imaginary part of $\mathrm{Zr}$ is negative, signifying the reduction in the overall inductance. Also note the increase in overall resistance due to the real part of Zr, yields an additional energy loss. From Eq. (1) and Eq. (2), it can be easily shown that the effect of $Z_{R}$ on $Z_{\text {input }}$ diminishes as $R_{2}$ approaches infinity. Our goal to reduce the effects of the induced coupling was to drive $R_{2}$ towards infinity.

To impede the eddy current flow in the other insulation layers and the associated emf, a new MLI layer was designed to include a staggered pattern of small copper traces. The prototype used for our test and evaluation is shown in Fig. 17. The traces were $2.54 \mathrm{~cm} \times 0.1 \mathrm{~cm}$. The separation between adjacent traces was $0.13 \mathrm{~mm}$. The design concept predicted the individual trace element would provide minimum surface area for the flow of eddy currents, while collectively providing a comparable surface emissivity $(\varepsilon)$ with that of the conventional solid sheet over the same per unit square area. For this evaluation the new effective surface area was $89 \%$ copper and $11 \%$ Kapton. The original material has an effective surface emissivity of 0.10 , while the new pattern provides an effective emissivity of 0.18 . With the use of improved manufacturing techniques, the design would be capable of achieving $95 \%$ coverage by providing smaller separations between traces.

Results of using the new MLI layer design on individual sensors are shown in Fig. 18. Twelve layers of the new MLI were individually placed on top of sensors having baseline frequencies of approx. 10.4 MHz. After each layer, the sensor response frequency was measured. The MLI was not directly adhered to the sensor, but allowed to contact the non-conductive Kapton surface of the sensor. The addition of the first layer created a $6 \mathrm{MHz}$ decrease in the sensor resonant frequency. The next three layers combined resulted in an additional decrease of $1 \mathrm{MHz}$. The remaining 8 MLI layers combined reduced the frequency by less than $0.5 \mathrm{MHz}$. These tests were performed on 3 sensors. The testing leads to the conclusion that sufficient numbers of patterned MLI layers could be utilized in applications with the sensors presented in this paper to achieve the same thermal efficiency as would be required with conventional MLI layers.

Results of using the new MLI layer design on top of the damage detection array are shown in Figs. 19 and 20. In Fig. 19 are responses from a undamaged array and an array that has a $4 \mathrm{~cm}$ by $4 \mathrm{~cm}$ hole in its center sensor. The lowest baseline is approximately $8.8 \mathrm{MHz}$ for the undamaged array. When the middle sensor is damaged with a 2.9 $\mathrm{cm}$ by $2.6 \mathrm{~cm}$ hole, the lowest response resonant that is at least $10 \mathrm{dBm}$ higher than the noise is at $11.4 \mathrm{MHz}$. This comparison was repeated with two of the new MLI layers placed on top of the array. Undamaged, the lowest response frequency was $3.5 \mathrm{MHz}$. A lowest response frequency of approximately $7.7 \mathrm{MHz}$ was measured for an array having a damaged sensor. The results of using the new MLI design on the damaged and undamaged array sensors shows that thermal insulation system (Sensor array layer and new design of the other layers to reduce the effect of eddy currents) presented in this paper is capable of providing damage detection to the thermal insulation of an inflatable habitat.

\section{Conclusions}

A method for using the multi-layer insulation of an inflatable habitat to detect and locate micrometeorite penetration to the habitat layers has been presented along with test results that validate the use of the concept. The method is facilitated by depositing specific geometric patterns of reflective electrically conductive material on the insulation layers. The layer used for damage detection has arrays of spirals. When exposed to oscillating magnetic fields, the spirals become electrically active and respond with their own magnetic fields. The response resonant frequencies and magnitude are dependent upon whether the sensors (i.e., the spirals) have been damaged. The 
sensors are electrically open circuits with no electrical connections. Because the sensors have no electrical connections, a potential source of failure has been eliminated. The other insulation layers have a brick pattern that reduces the amount of eddy currents created when the sensors respond with their own magnetic fields. With the brick patterned layers, the sensors' response can permeate the insulation layers so that the sensor can be interrogated.

Testing of individual $15.25 \mathrm{~cm}$ by $15.25 \mathrm{~cm}$ sensors demonstrated that each sensor can be used to identify at least three damage locations per sensor. The sensors were damaged by placing holes from $0.2 \mathrm{~cm}$ to $4.0 \mathrm{~cm}$ in them. In other tests, sensors were sequentially damaged with up to three $0.2 \mathrm{~cm}$ holes. In all tests, the sensor response frequency shifted and established a new measurement baseline. Two sensor arrays formed from 9 sensors (3 rows by 3 columns) were tested using the hypervelocity impact facility at White Sands Test Facility. Each array was part of a testbed to examine different damage detection concepts. Each array had 5 metal projectiles of 1-3.6 mm dia. propelled at them at speeds ranging from $6.7-7.1 \mathrm{Km} / \mathrm{s}$. One array was placed on the outer habitat shell layer and the other was placed one layer inboard. The projectiles produced five holes less than $0.9 \mathrm{~cm}$ on the array on the outer layer. The array on the inboard layer had three locations with the following damage 1) a $6.3 \mathrm{~cm}$ rip 2) a hole $5.6 \mathrm{~cm}$ by $0.8 \mathrm{~cm}$ with a $4.6 \mathrm{~cm}$ rip and smaller rips 3 ) a $1.8 \mathrm{~cm}$ by $1.8 \mathrm{~cm}$ hole with a $3.4 \mathrm{~cm}$ rip and a $3.6 \mathrm{~cm}$ rip. In all cases, every sensor on both arrays could still be powered, interrogated and used to detect subsequent damage.

The inductive coupling provided some means of telemetry because damage to one sensor would affect the response of the other sensors. To identify a damage event, only one sensor needed to be interrogated. This method of telemetry was used during the hypervelocity impact tests to provide real-time indications of damage to the array. Examination of sequential damage to the array led to two methods for identifying which sensor has been most recently damaged. The sensor that was most recently damaged has the highest change in the area under its response curve and the largest shift to its resonant frequencies. The first method to locate damage is to identify which sensor had the largest Euclidean norm derived using the change in response magnitudes. The sensor with the largest norm is the sensor that has been damaged by the most recent damage event. The second method is to identify the sensor having the largest shift in its resonant frequencies.

To serve as a method of thermal protection, the insulation layers must have minimal radiative emittance. The modifications to any thermal array design should negligibly affect the accumulative emittance of all layers. The sensor array had a $6 \%$ higher emittance than a continuous layer of reflective metal. Using the brick pattern of reflective material for the other insulation layers, produced an emittance that was $11 \%$ higher. An array using the damage detection layer and the new brick patterned insulation layers presented in this paper should have total emittance for the entire array slightly higher than an array that does not use this design. One or two additional layers should result in the same level of emissivity. Comparison of the frequency response using the new multi-layer insulation system (damage detection array + brick patterned insulation layers) with an undamaged sensor in the array to using the system with a damaged sensor in the array showed the system was capable of both damage detection and location.

In future work, we hope to examine larger arrays to address scalability of this method. Because no electrical connections are used, the method should be scalable. Another goal of future work is to develop the sensor response frequency range so that when two or more sensors are used together in close proximity, the inductive coupling as a means of telemetry can be better exploited. Other future work would be developing rules for tailoring sensor geometry to make the sensors in the outer shell layers more responsive to smaller damage.

The objective of this study was to develop a means of detecting micrometeorite damage and if possible to use the thermal protection layers for the detection. The results of this study have demonstrated thermal protection and damage detection can be derived from the same construct. To achieve the design of the multifunctional assembly, a new class of sensors - open-circuits having no electrical connections - was developed. The manufacturing process to make the sensors is just the deposition of an electrical trace. That process is one of many manufacturing processes necessary to manufacture other sensors. Many measurement applications that use magnetic field response sensing with the inductor electrically connected to the capacitor in a closed circuit can be replaced using the simpler open circuit design that has no electrical connections as presented in this paper. To date, the new class of sensor is being adapted to a variety of measurements such as liquid level, temperature, displacement, wheel speed, chemical and tamper detection. 


\section{Acknowledgments}

The authors thank the following for their support and help: Karen M Rodriguez, NASA JSC White Sands Test Facility; Mr. Keith Murray and Mr. Dennis Bushnell of NASA Langley Research Center; Mr. George Studor, NASA JSC and Dr. Erik Brandon, NASA JPL.

\section{References}

${ }^{1}$ Kennedy, K. J., "Lessons from TransHab - An Architects Experience,”AIAA Space Architecture Symposium, Oct 10-11, 2002, AIAA 2002-6105.

${ }^{2}$ Benaroya, H., "An Overview of Lunar Base Structures: Past and Future,”AIAA Space Architecture Symposium, Oct 10-11, 2002, AIAA 2002-6113.

${ }^{3}$ Moore, G. T. and Rebholz, P. J., “Aerospace Architecture: A Comparative Analysis of Five Lunar Habitats," 1992 Aerospace Design Conference, Feb 1992, Irvine, CA, 2002, AIAA 92-1096.

${ }^{4}$ Bille, M., "The Satellites of Sergei Korolov," 41 Aerospace Sciences Meeting and Exhibit, January 2003. Reno, Nevada, AIAA 2003-660-386.

${ }^{5}$ Bille, M., "Project Vanguard: Setting the Record Straight," 41 Aerospace Sciences Meeting and Exhibit, January 2003. Reno, Nevada, AIAA 2003-657-772.

${ }^{6}$ Orrok, G.T., "The Meteoroid Hazard to Project Apollo, NASA CR 125273, Oct 1962.

${ }^{7}$ Gilmore, D. G., "Spacecraft Thermal Control Handbook,"The Aerospace Press, El Segundo, CA, 2002, pp 161-205

${ }^{8}$ Woodard, S. E., and Taylor, B. D. "Wireless Sensing Using Open-Circuit Electrically Conductive Spiral-Trace Sensor," Filed as U. S. Patent Application Feb 5, 2007 and filed on Feb 6, 2007 for international protection via the Patent Co-operation Treaty with the World Intellectual Property Organization.

${ }^{9}$ Woodard, S. E., Jones, T. W., Taylor, B. D. and Shams, Q. A., "Damage Detection/Location system Providing Thermal Protection," Filed as U. S. Patent Application Feb 5, 2007 and filed on Feb 6, 2007 for international protection via the Patent Cooperation Treaty with the World Intellectual Property Organization.

${ }^{10}$ Woodard, S. E., Taylor, B. D. , Shams, Q. A. and Fox, R. L., "Magnetic Field Response Measurement Acquisition System," NASA Technical Memorandum 2005-213518, February 2005.

${ }^{11}$ Seitz, M. and Roeber, M., "Squeeze More Performance Out of Toroidal Inductors," Power Electronics Technology, Aug 2005, pp 32-33.

${ }^{12}$ White, Harvey E., Modern College Physics, 6th Ed. Van Nostrand Reinhold, 1972. 


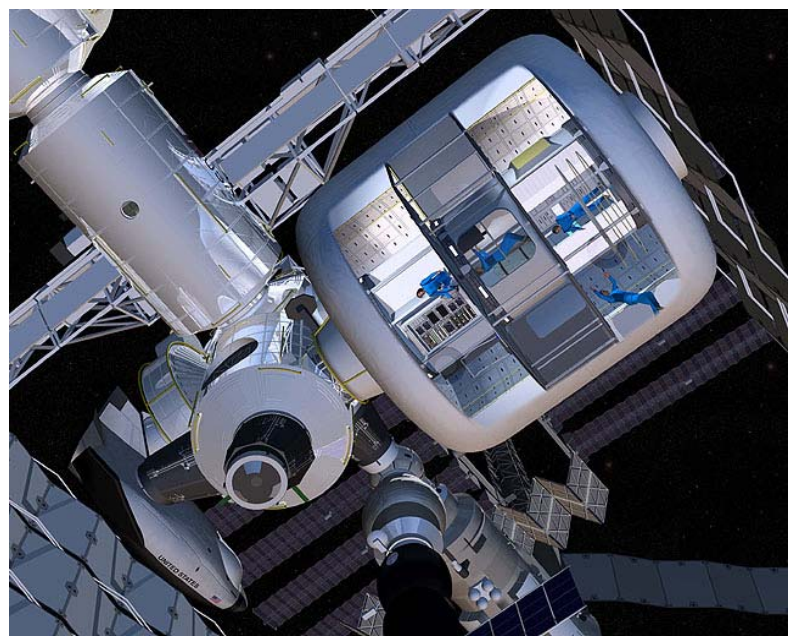

Figure 1. TransHab inflatable/deployable habitat module

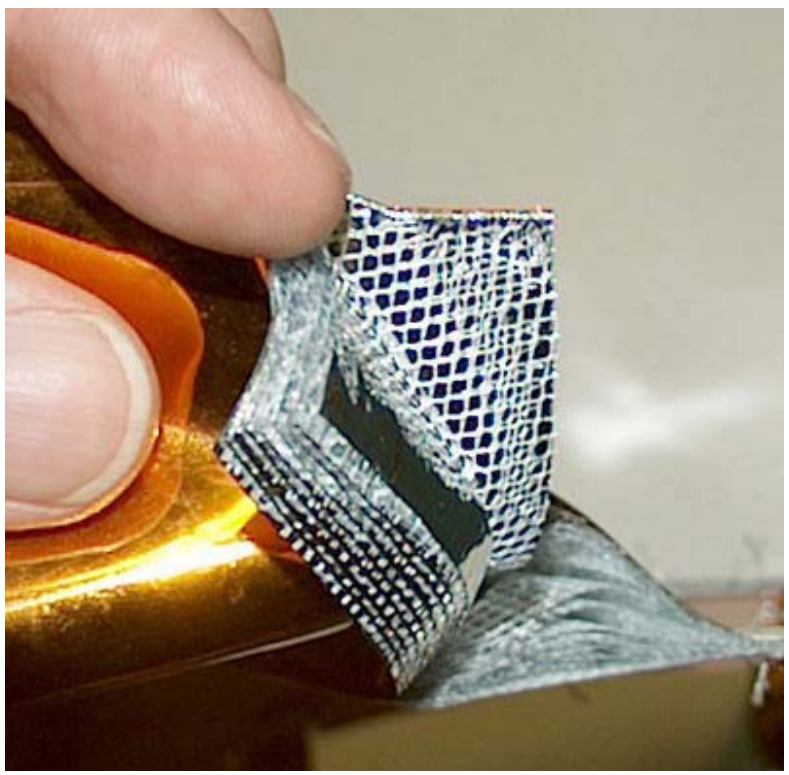

Figure 2. Multi-layer Insulation (MLI) construction 


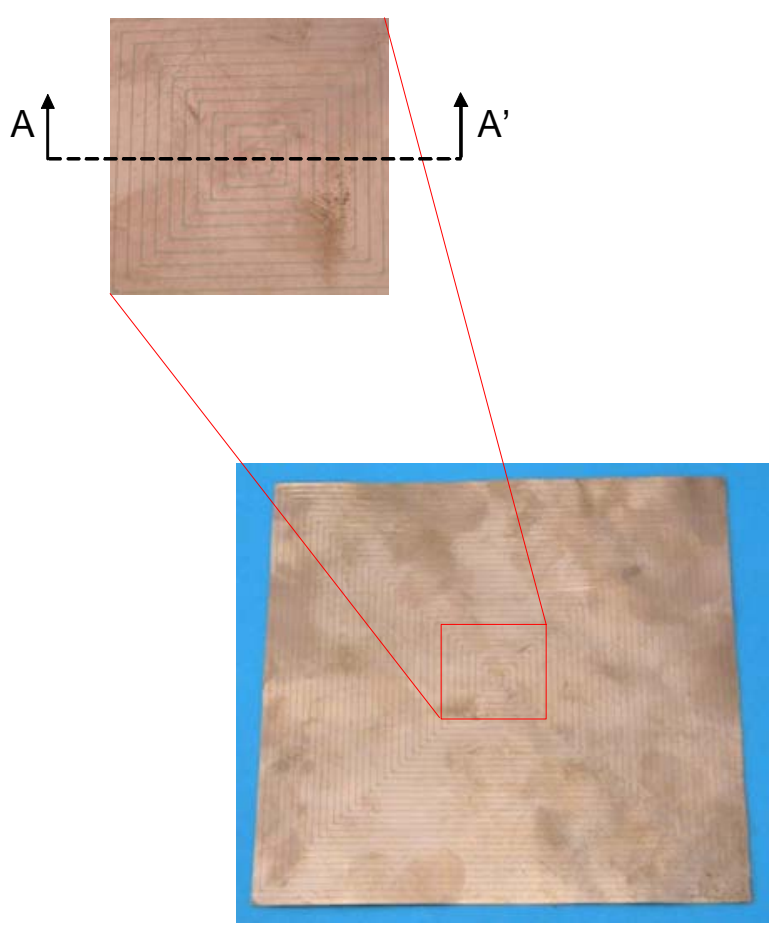

a) Sensor and enlarged view of trace

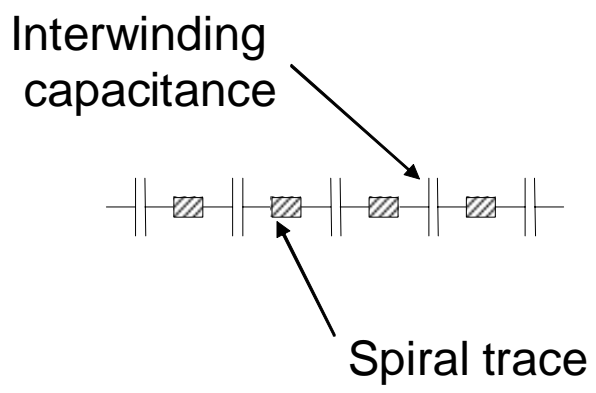

b) Cross Section A-A' schematic

Figure 3. Open-circuit electrically conductive spiral trace sensor used for damage detection

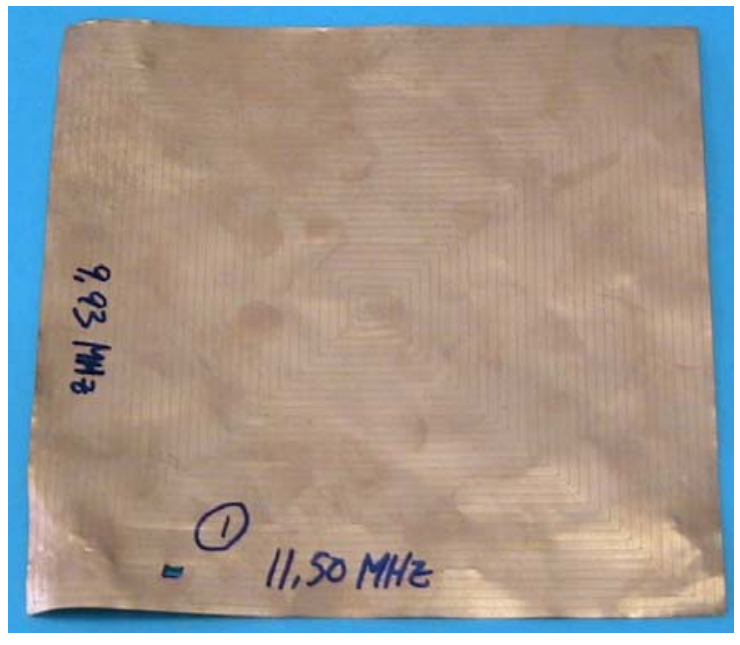

a) Damage to sensor with one $2 \mathrm{~mm}$ hole

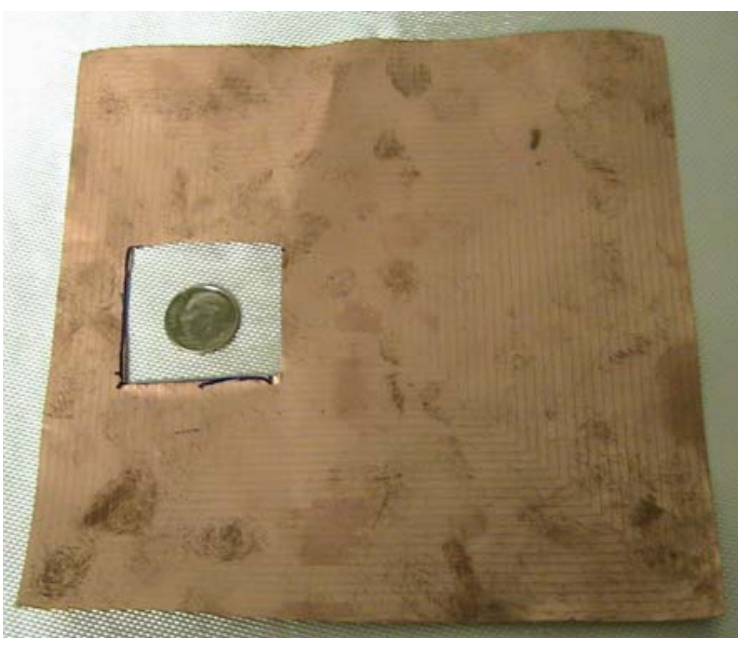

b) Damage to sensor with one $2.5 \mathrm{~cm}$ hole

Figure 4. Sensors damaged with single hole 


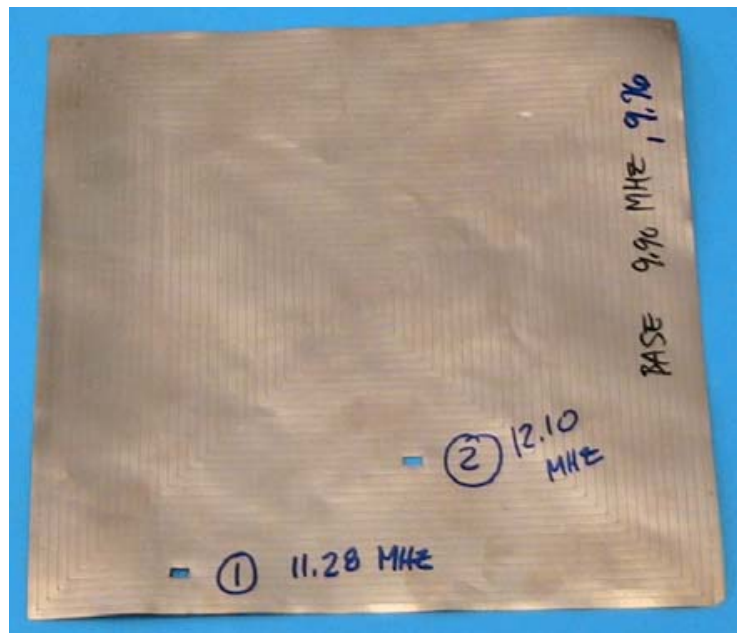

a) Sensors with two $2 \mathrm{~mm}$ punctures Figure 5. Measured frequencies of dam

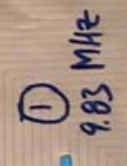 \\ (2) \\ $12.17 \mathrm{mHz}$}

$10.39 \mathrm{MHz}$

b) Sensors with three $2 \mathrm{~mm}$ punctures

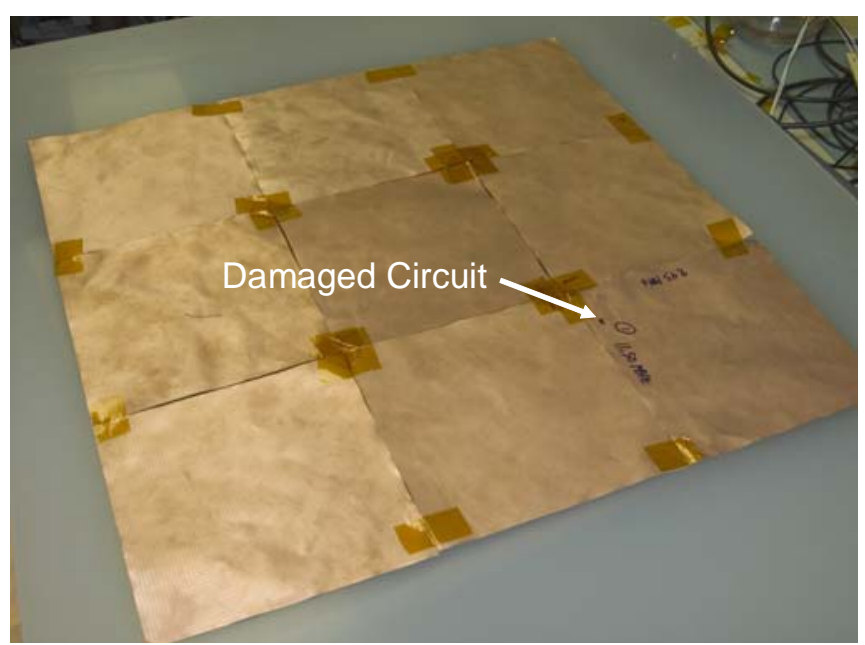

a) Array used for preliminary testing

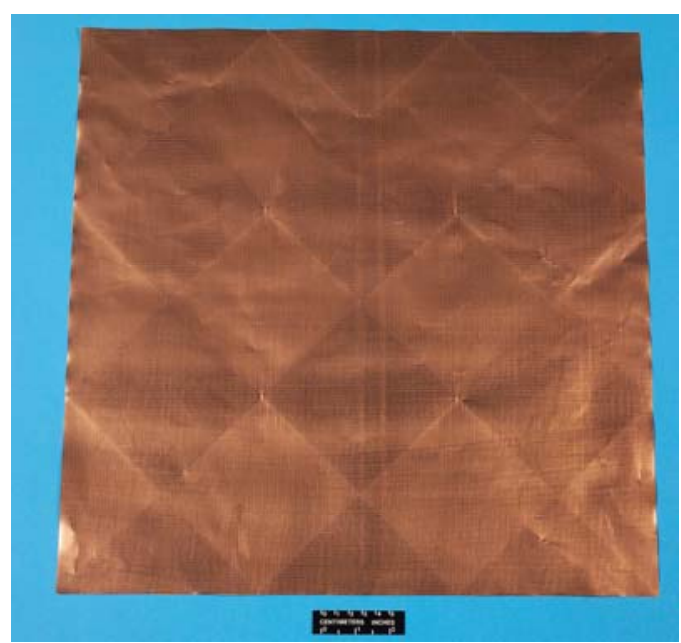

b) Array used for Hypervelocity Impact Testing

Figure 6. Damage detection array ( 3 sensors $\mathrm{x} 3$ sensors) 


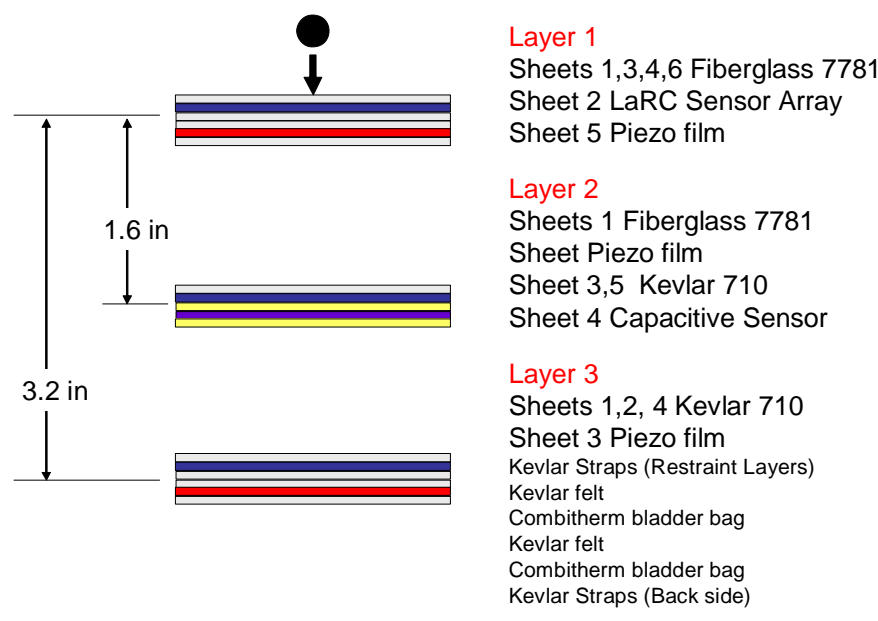

a) Schematic of first hypervelocity impact test article layers

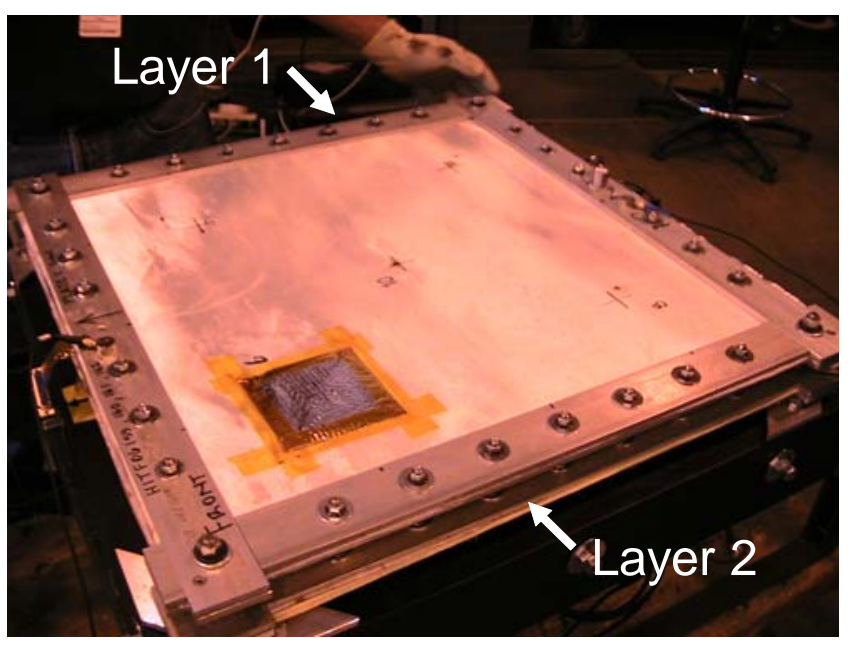

b) First hypervelocity impact test article layers

Figure 7. Test article and schematic of first test article layers 


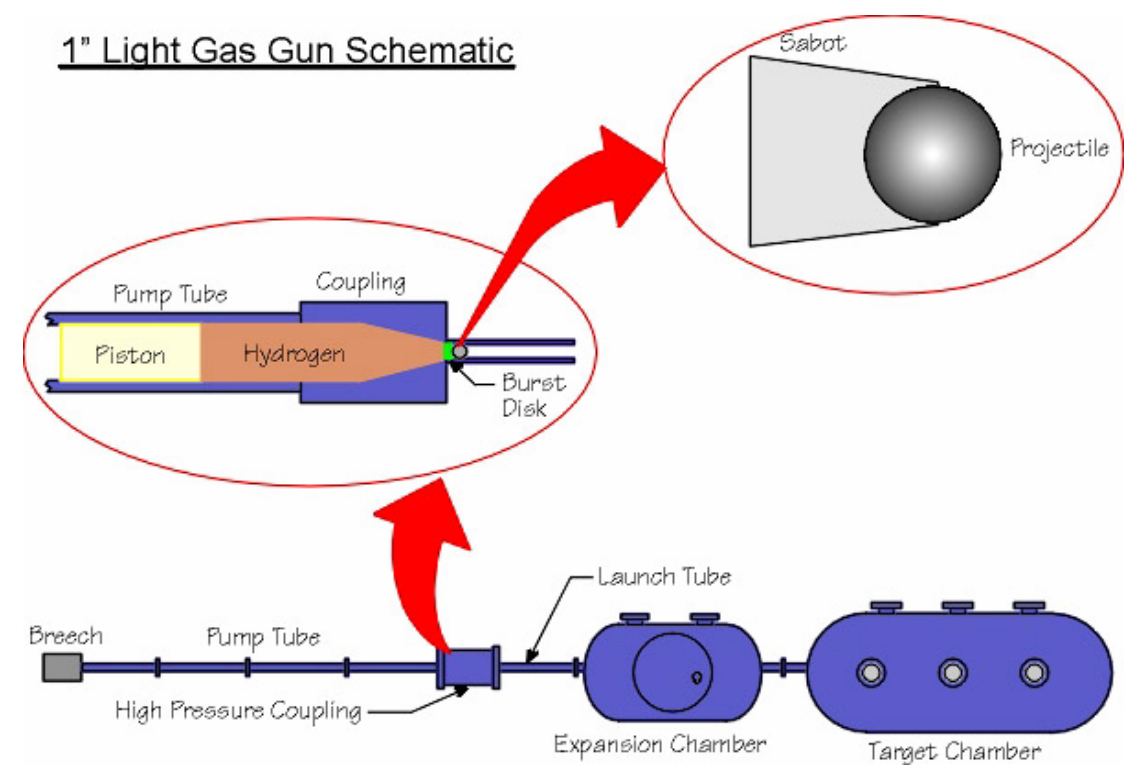

a) Schematic of hypervelocity test chamber.

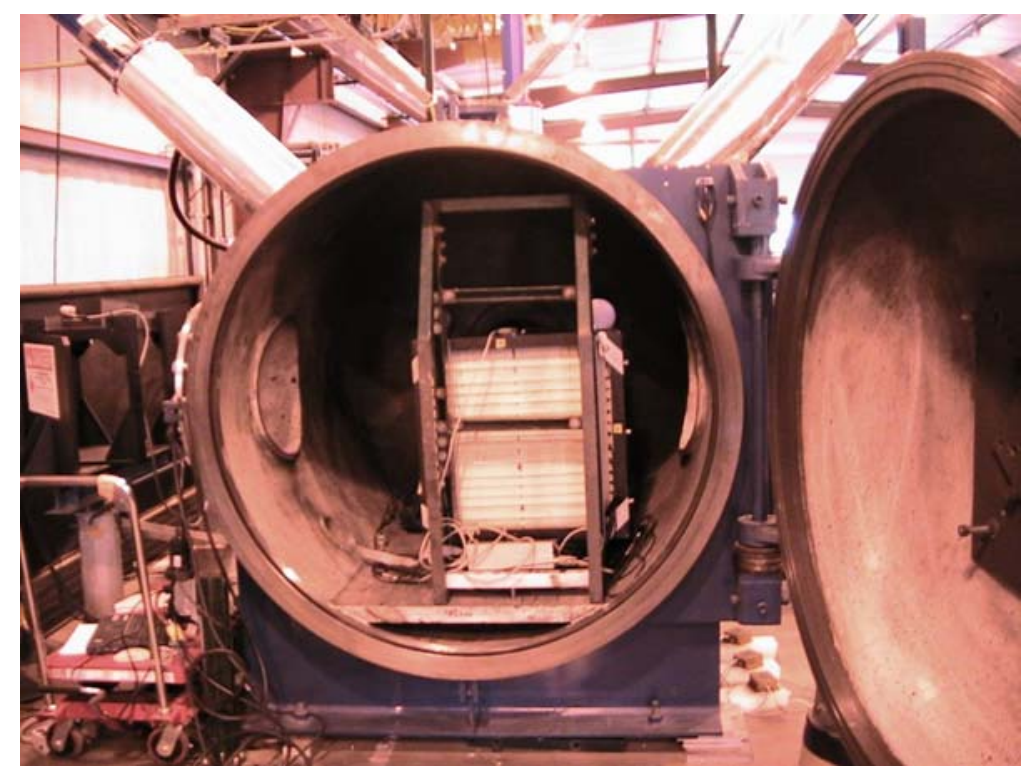

b) Test article mounted to support in target chamber

Figure 8. Hypervelocity test chamber and test article inside target chamber. 


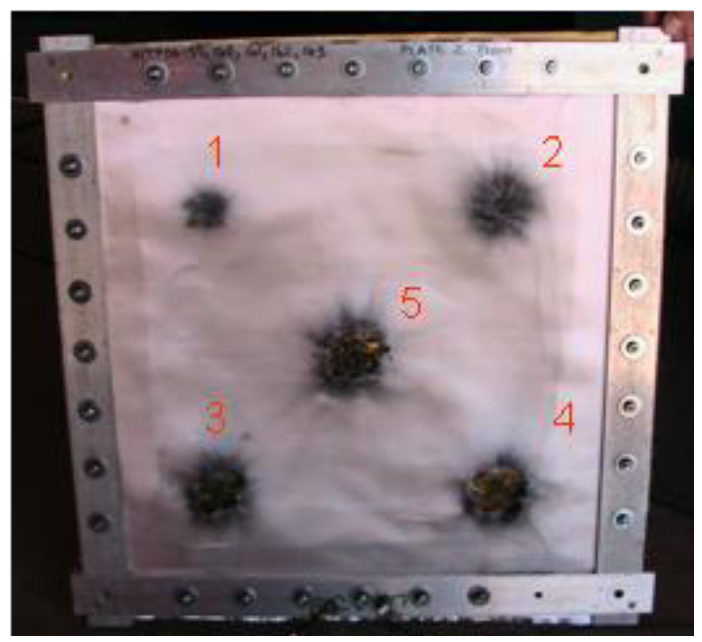

a) First test article having sensor array No. 2 after impact tests1-5

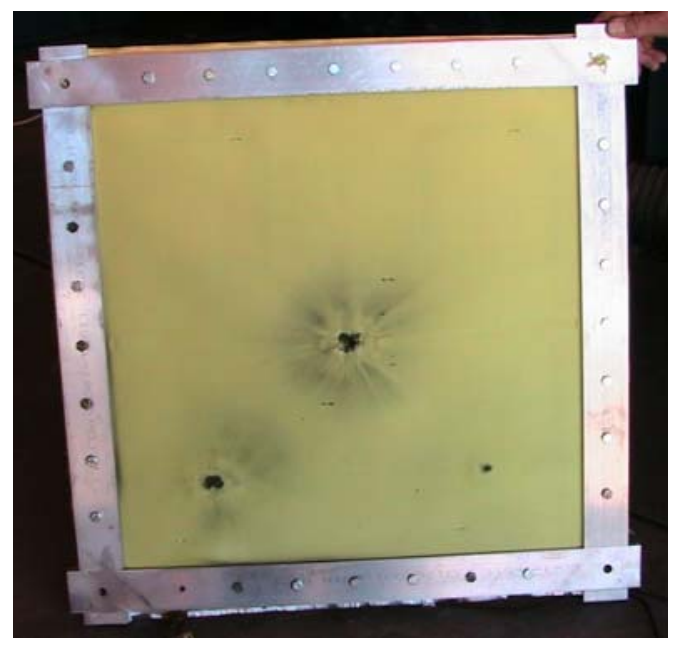

b) Second test article having sensor array No. 5 after impact tests 6-10

Figure 9. Damage to test articles. Impact positions are annotated on 9a.

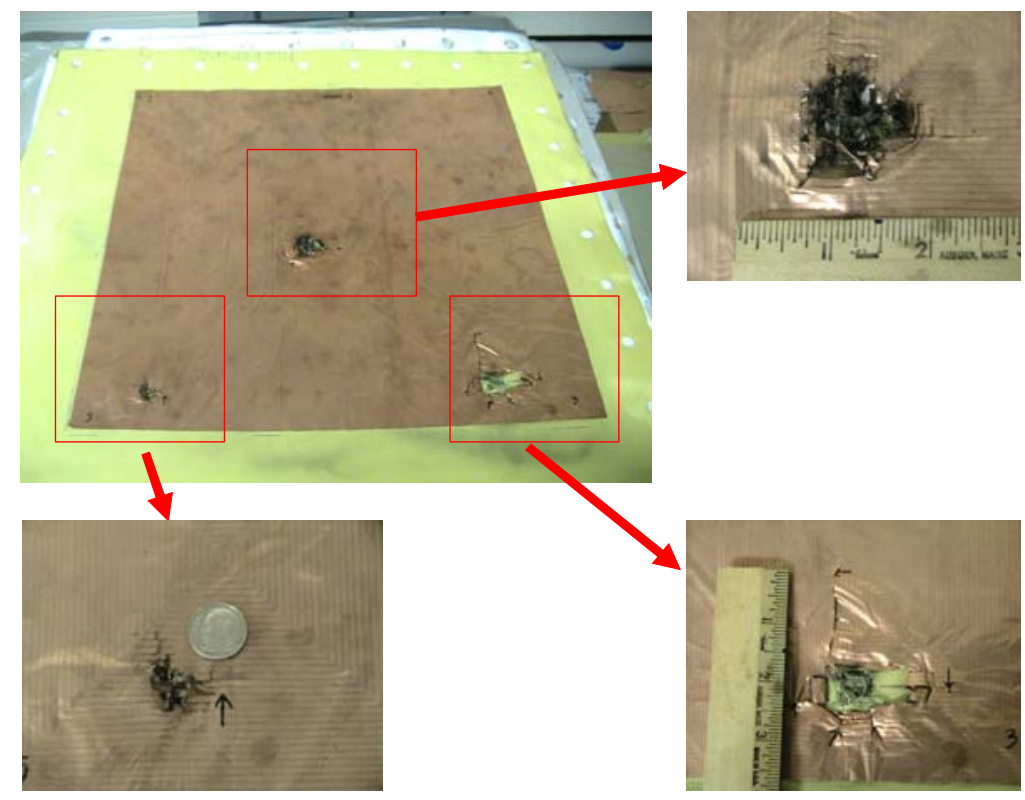

Figure 10. Magnetic field response sensor array after completion of tests $6 \mathbf{- 1 0}$. 


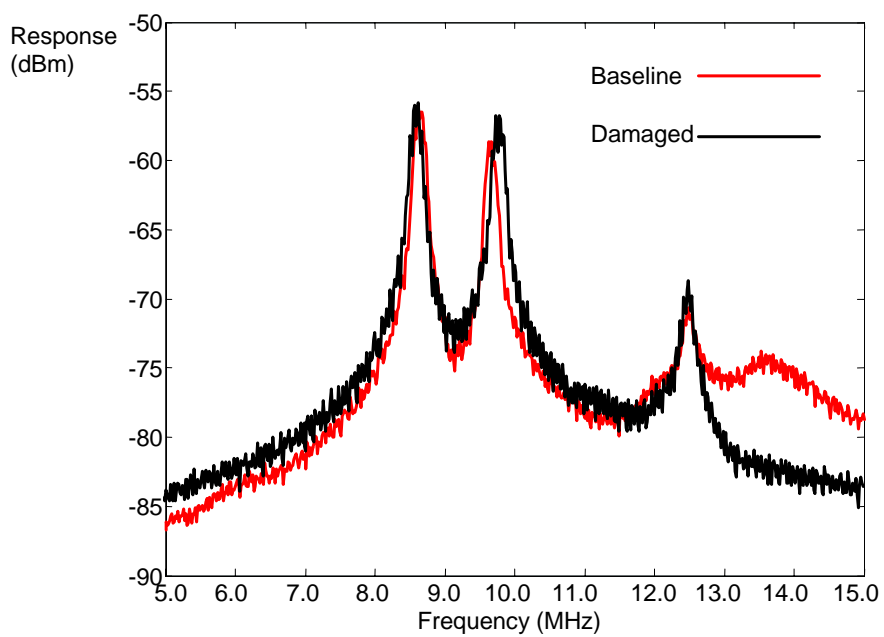

Figure 11. Sensor 9 of Array 2 response change due to hypervelocity impact tests 1 - 5.

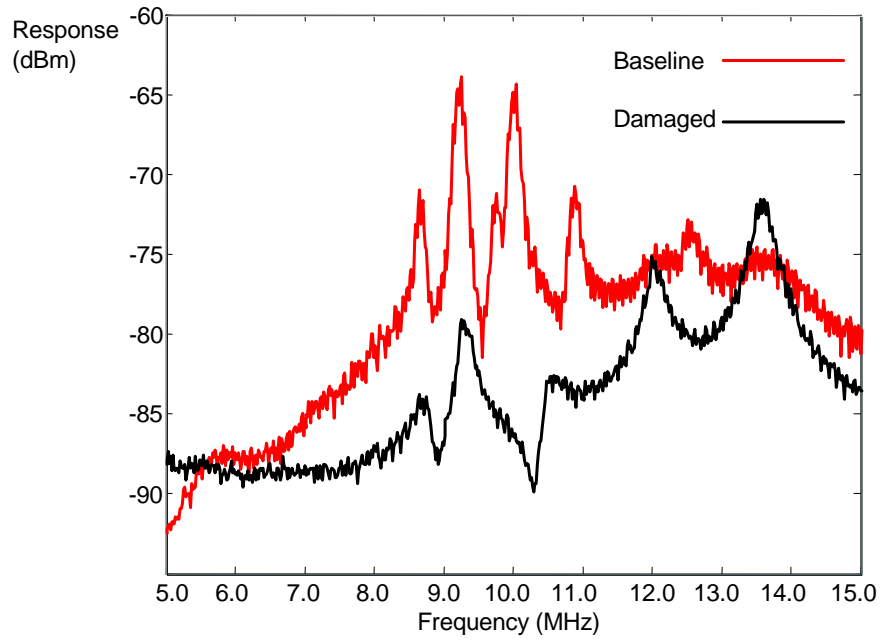

Figure 12. Sensor 3 of Array 5 response change due to hypervelocity impact tests 6 - 10. 


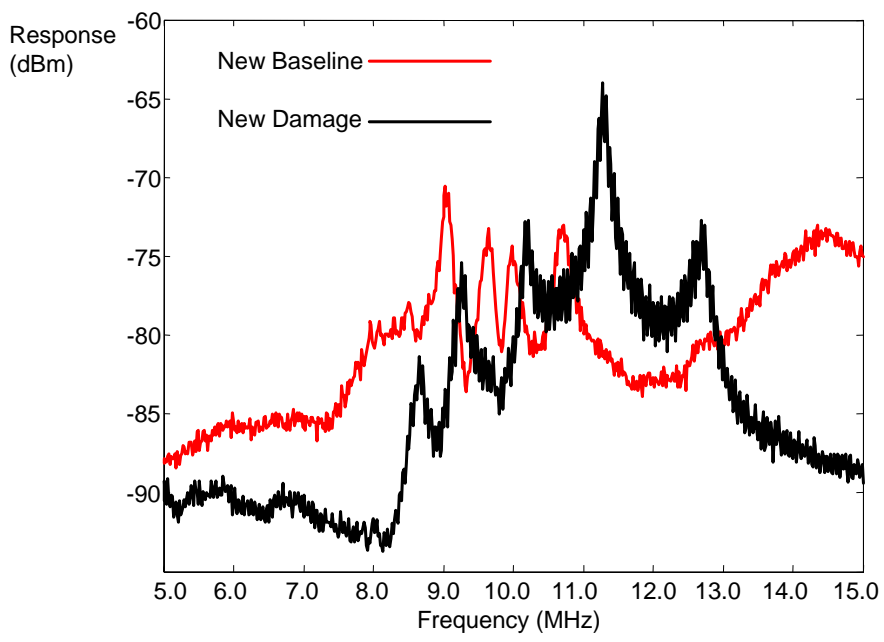

a) Sensor 5 response without any damage and response after it has been damaged.

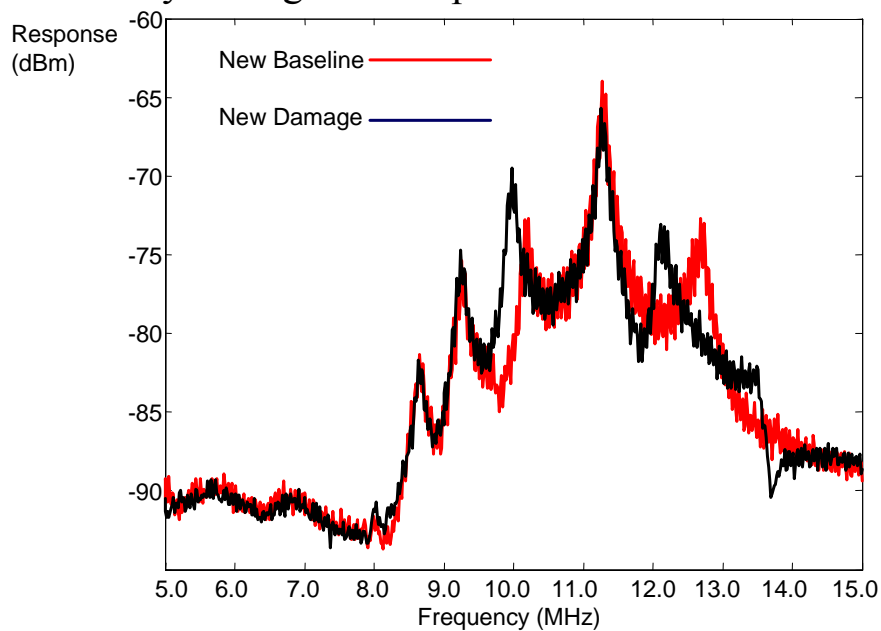

b) New sensor 5 baseline after it has been damaged and response of sensor 5 after sensor 3 has been damaged.

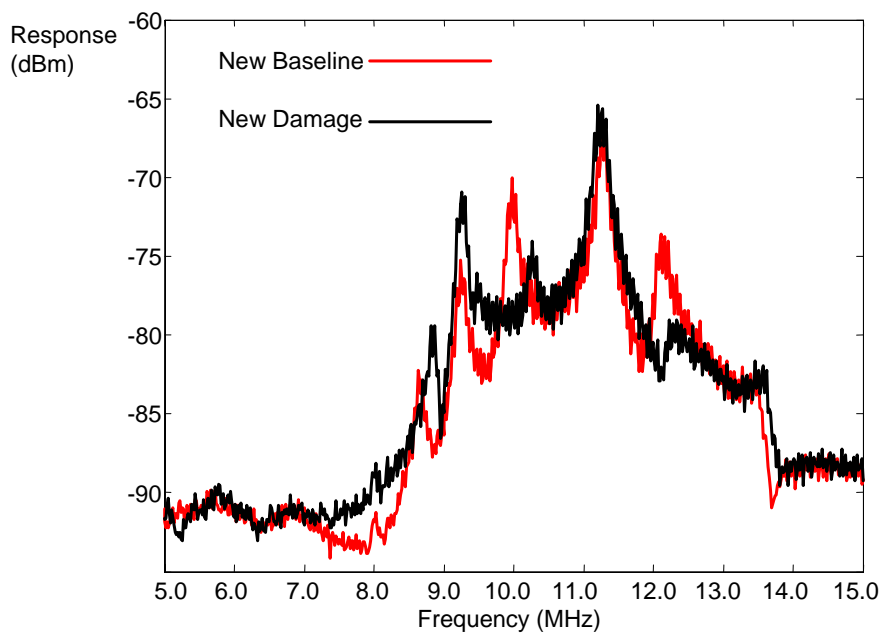

c) New sensor 5 baseline after sensor 3 has been damaged and response of sensor 5 after sensor 9 has been damaged.

Figure 13. Sequential damage to Array 6 measured at sensor 5. Damage occurs to sensors 5, 3 and 9 in that respective order. 


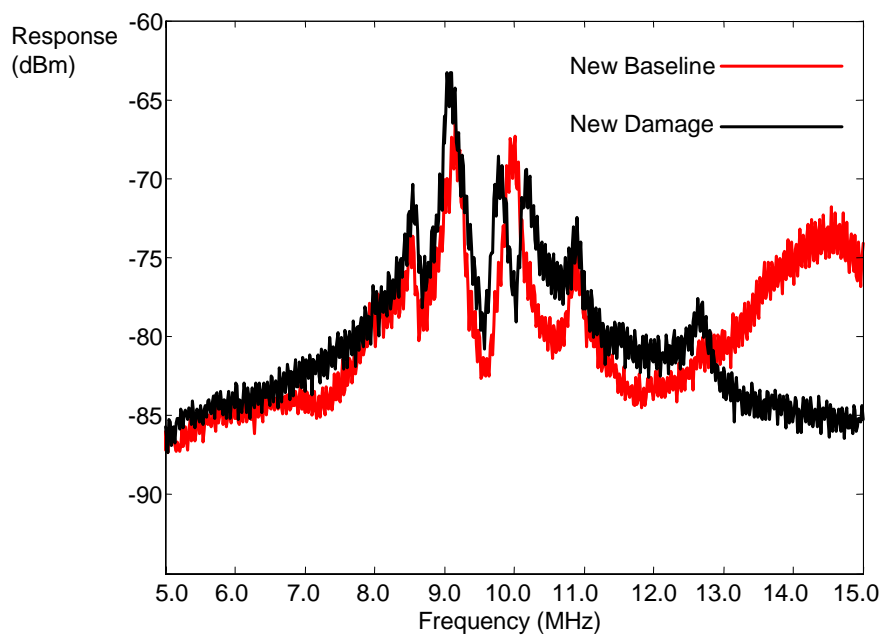

a) Sensor 3 response without any damage and response after sensor 5 has been damaged.

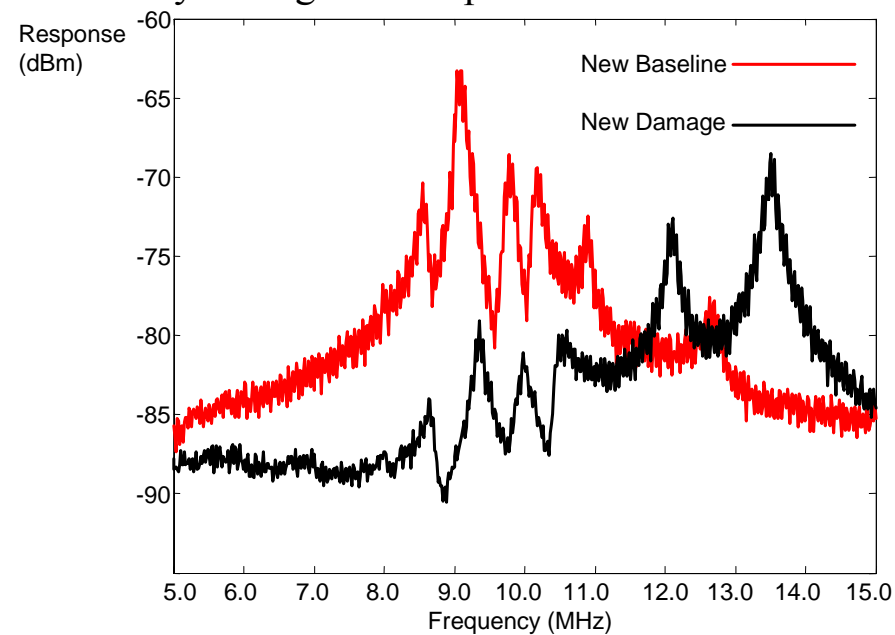

b) New sensor 3 baseline after sensor 5 has been damaged and response of sensor 3 after it has been damaged.

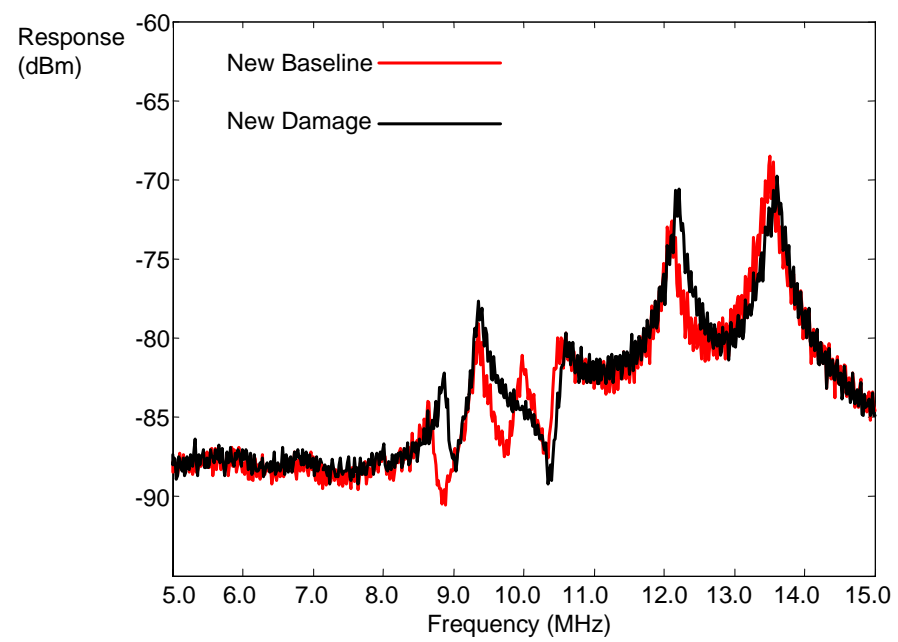

c) New sensor 3 baseline after it has been damaged and response of sensor 3 after sensor 9 has been damaged.

Figure 14. Sequential damage to Array 6 measured at sensor 3. Damage occurs to sensors 5, 3 and 9 in that respective order. 


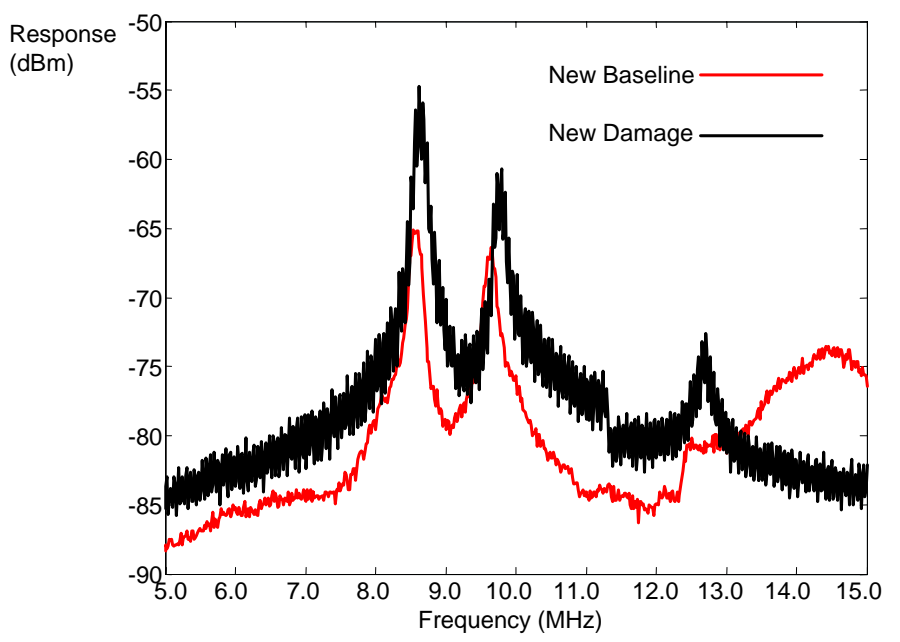

a) Sensor 9 response without any damage and response after sensor 5 has been damaged.

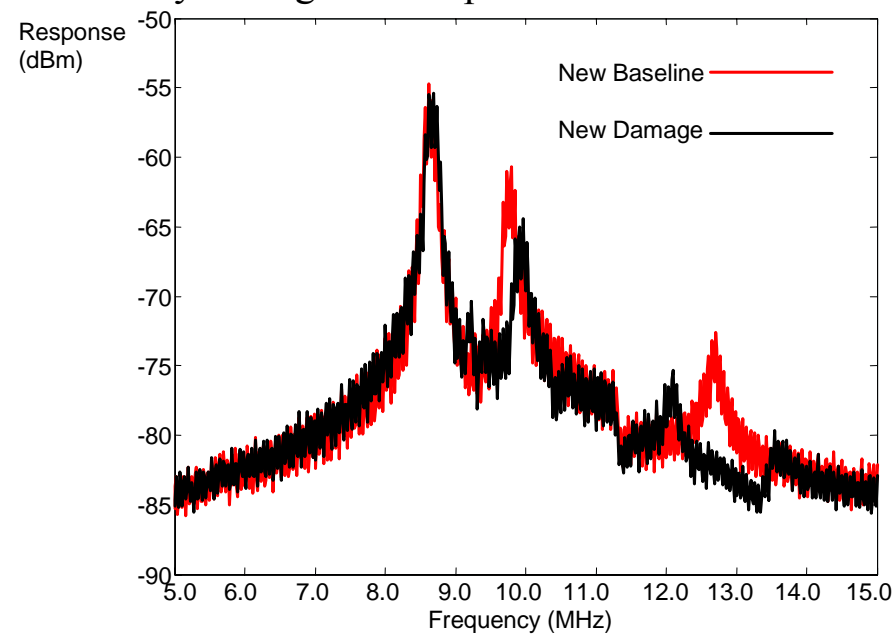

b) New sensor 9 baseline after sensor 5 has been damaged and response of sensor 9 after sensor 3 has been damaged.

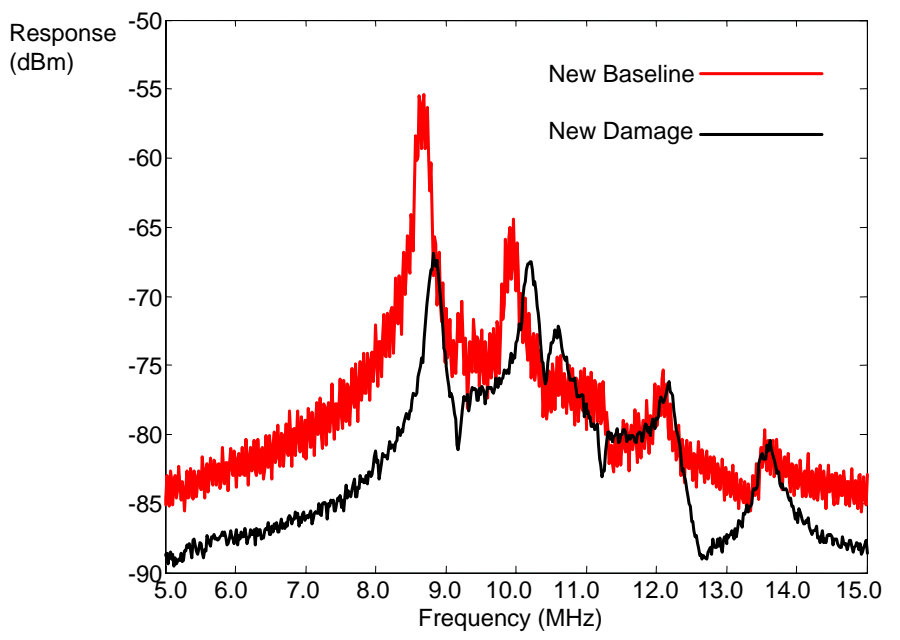

c) New sensor 9 baseline after sensor 3 has been damaged and response of sensor 9 after it has been damaged.

Figure 15. Sequential damage to Array 6 measured at sensor 9. Damage occurs to sensors 5, 3 and 9 in that respective order. 


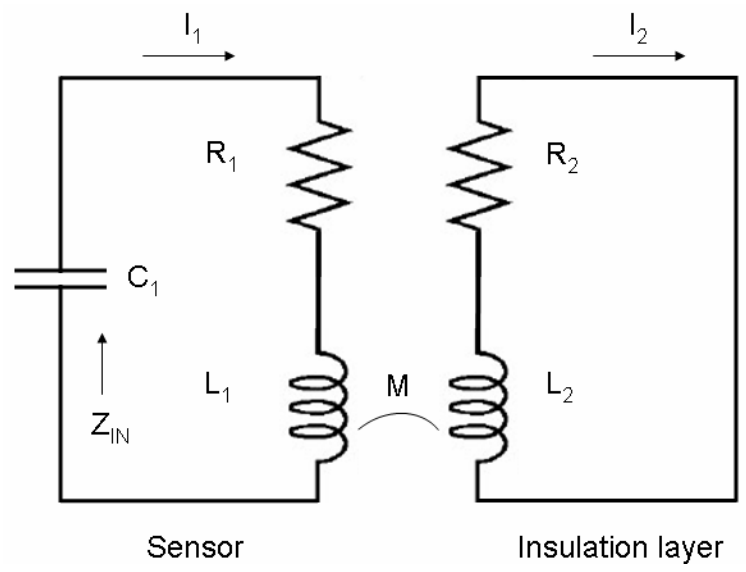

Figure 16. Model of effect of insulation layer with a continuous metallic covering on sensor response

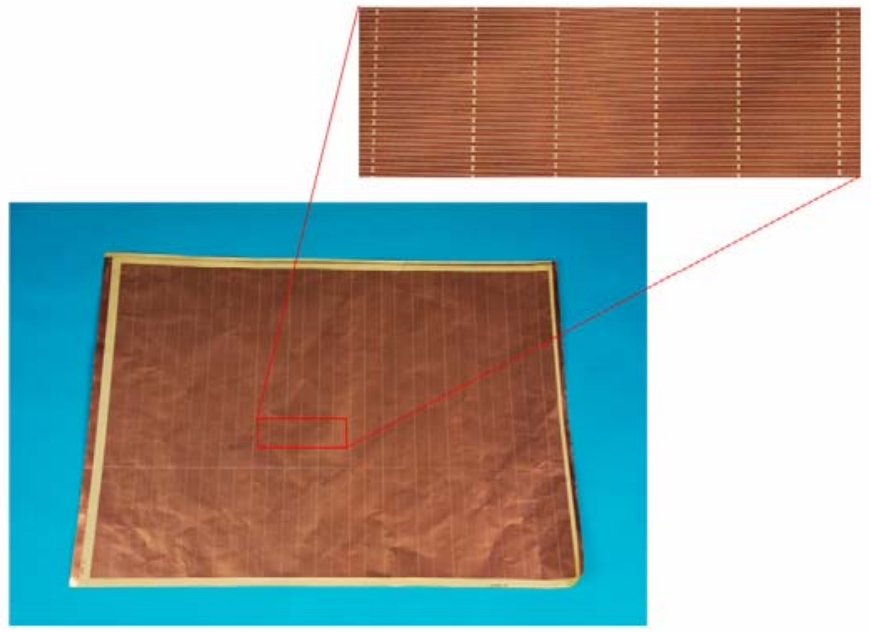

Figure 17. New MLI layer design that reduces development of Eddy currents.

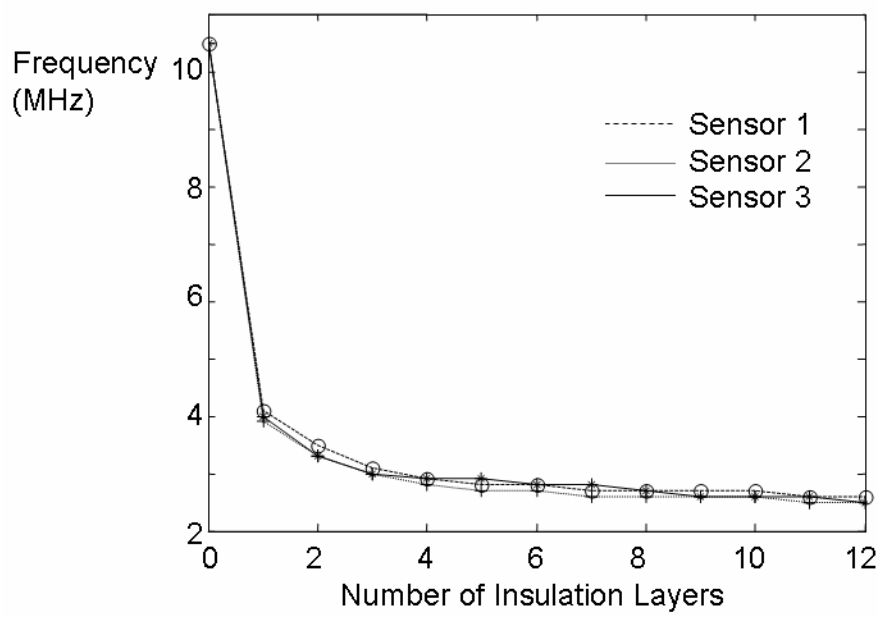

Figure 18. Response frequency shift with addition of insulation layers 


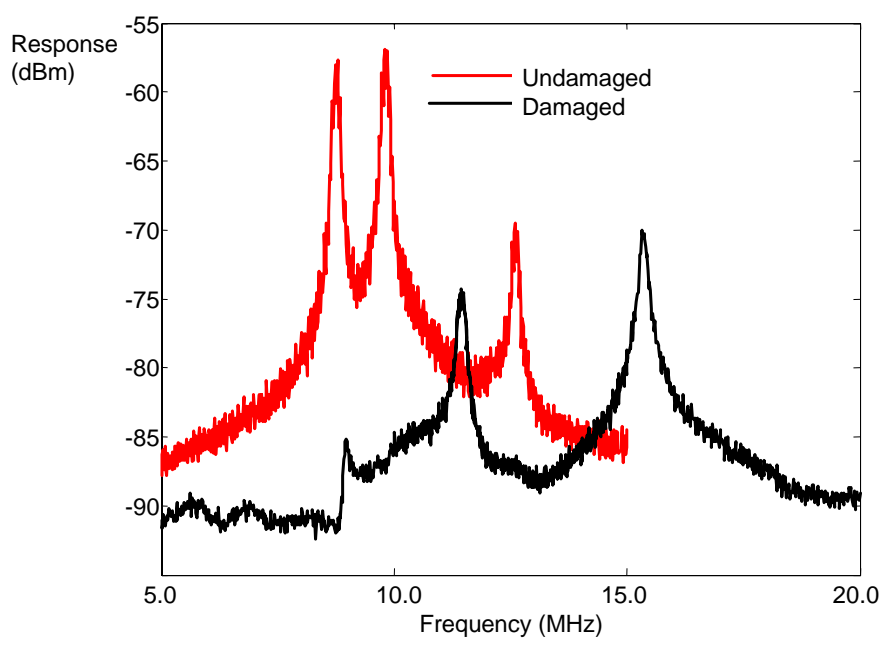

Figure 19. Response measurement at Sensor 9 of Array 4 before and after damage to sensor 9 .

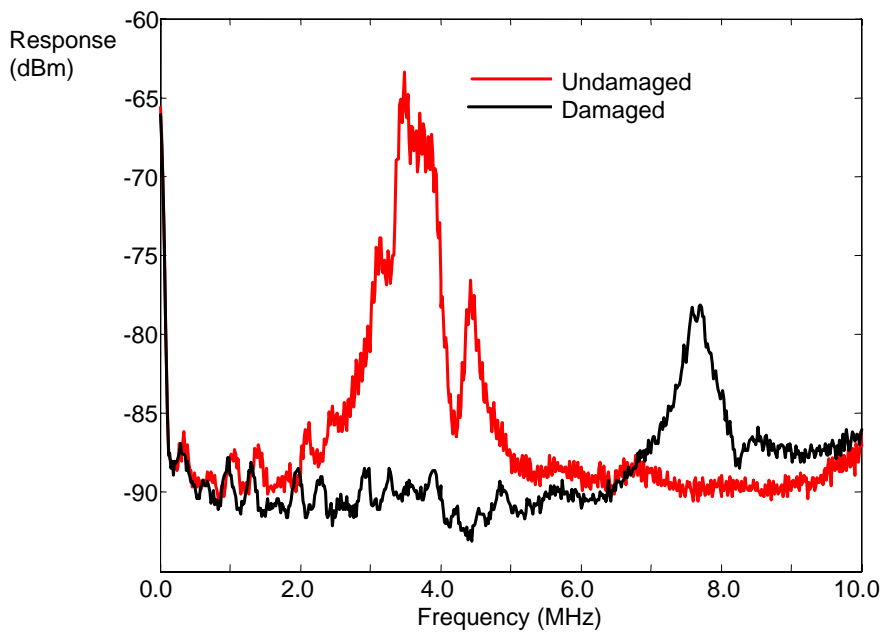

Figure 20. Response measurement at Sensor 9 of Array 4 with two insulation layers before and after damage to sensor 9 . 